complications of cirrhosis) are being detected, allowing patient care to be adjusted in light of these findings. As the service has developed, referral numbers have increased due to increased awareness and positive reputation developed by the service. The procedures have improved in quality, with a rising D2 intubation rate and a falling duration of procedure. These data demonstrate that an endoscopy service can be successful delivered, using innovative teaching methods and ongoing support delivered by a UK team. The endoscopists are now delivering training to new trainees, with a view to self sufficiency in training and expansion to other areas in Sierra Leone.

\section{OTH-07 IDENTIFICATION OF IBD COHORTS FROM LINKED ENDOSCOPY AND HISTOLOGY REPORTS USING NATURAL LANGUAGE PROCESSING}

1Jonathan Brown*, '2Sebastian Zeki. 'Gloucestershire Hospitals Nhs Trust, Gloucester, UK; ${ }^{2}$ Gastroenterology Data Sciences Institute, St Thomas' Hospital, London SE1, UK

\subsection{6/gutjnl-2019-BSGAbstracts.426}

Introduction Patients with inflammatory bowel disease (IBD) are likely to undergo multiple lifetime endoscopic procedures which generate histopathological reports. Managing these patients requires clinicians to derive a phenotypic overview from numerous episodes and diverse sources which can be time consuming, incomplete and subjective. We set out to evaluate the potential for a computer to extract phenotypic parameters from a series of linked histopathology and endoscopy reports to characterise an IBD cohort.

Methods 118,108 lower GI endoscopic procedure reports (200-017) and 62,051 lower GI histology reports (200-017) from GRH were imported into an SQL database. Unique patient identification numbers from the merged dataset were replaced with 128 bit hexadecimal GUIDs and all patient identifiable information subsequently stripped from the data tables (Service Evaluation Project 8622).

Text processing was undertaken in Python pandas dataframes:

1) Import both datasets and separate all words by single space, convert to lower case, remove apostrophes

2) Correct spelling of key words using Levenshtein distance

3) Find regular expressions that match disease phenotypes

4) Exclude non-IBD colitis diagnoses

5) Exclude negated IBD diagnoses

6) Export tagged machine interpreted reports back to SQL database

7) Select 100 random reports for each IBD confirmed or negated diagnosis to validate against original text

8) Return to steps - to modify regular expression reference lists to improve sensitivity and specificity.

Results The following results were obtained after multiple validation cycles initially based on an empiric regular expression dataset.

\begin{tabular}{lll}
\hline & Sensitivity & Specificity \\
\hline Procedure indication: Previously known IBD colitis & $100 \%$ & $100 \%$ \\
Procedure indication: Previously known Crohn's & $100 \%$ & $100 \%$ \\
disease & & \\
Histopathological interpretation: IBD colitis & $100 \%$ & $98 \%$ \\
Histopathological interpretation: IBD Crohn's & $100 \%$ & $98 \%$ \\
\hline
\end{tabular}

Some caution is required in interpretation of the specificity of the Crohn's and ulcerative colitis histopathology reports. Many samples are described as showing features of both diseases and the final conclusion is given as a likelihood or unclassified. The specificities reported here are for all IBD and do not reflect a capacity to distinguish between the different types.

Conclusions The evolution of the disease characteristic regular expressions through repeated validation cycles has provided a powerful tool for the automated generation of IBD databases from text in semi-structured endoscopy and histology reports. The potential for the scheduling of surveillance and linkage to other systems, such as primary care prescribing, are obvious. Further development will include a more detailed phenotypic interpretation and computation of the histopathological certainty in distinguishing the types of IBD.

\section{OTH-11 INTELLIGENT LIVER FUNCTION TESTING (ILFT) IN ACTION}

${ }^{1}$ Emma Robinson*, ${ }^{2}$ Jennifer Nobes, ${ }^{1}$ Paul Brennan, ${ }^{2}$ Ellie Dow, ${ }^{1}$ John Dillon. ${ }^{1}$ University of Dundee, Dundee, UK; ${ }^{2}$ NHS Tayside, Dundee, UK

\subsection{6/gutjinl-2019-BSGAbstracts.427}

Background and aims Liver Function Tests (LFTs) are commonly requested and are abnormal in $20 \%$ of cases. Intelligent liver function testing (iLFT) aims to improve diagnostic proficiency and quality of investigation thereby reducing overall costs to practitioners and patients and subsequent secondary care referrals. Following a pilot trial, iLFT was rolled out across general practices in NHS Tayside from August 2018.

Method The automated iLFT algorithm uses the combination of clinical features (alcohol consumption, BMI and metabolic syndrome), diagnostic criteria for liver disease, an investigation ordering and reporting system, and the tracked blood sciences system to generate a diagnosis or descriptor of the abnormality with fibrosis staging.

Management plans are disseminated back to the GP, along with a recommendation for one of three outcomes; a) secondary care follow up for advanced liver disease or complex treatment, b) primary care follow up of early or simple liver disease, c) where a clear diagnosis is unclear; the GP receives staging and prognostic information including referral criteria. Results of iLFT in action, over 6 months were analysed.

Results 777 iLFT requests over 6 months to 31/01/19. 568/ 777 requests had at least 1 abnormal LFT triggering the iLFT cascade. $169(29.8 \%)$ were referred to secondary care, 399 (70.2\%) patients were investigated further or managed in primary care. The most common finding was isolated ALT elevation without fibrosis (24.8\%), followed by alcohol related liver disease without fibrosis(16.4\%) then non-alcoholic fatty liver disease with fibrosis (8.1\%) and elevated ALT and GGT without fibrosis (8.1\%). There were $2 \mathrm{HBV}$ infections, 8 HCV infections, 3 haemochromatosis diagnoses, $1 \mathrm{PBC}$ and 1 A1AT PiS variant (risk of lung/liver disease) and 17 carriers of A1AT S or Z variant.

Conclusion Using iLFT; serious liver disease has been detected and referred to secondary care. High numbers of LFTs have been investigated readily and management plans generated. Of all the patients with abnormal LFT results only a third have 
been referred to secondary care with two thirds being investigated or managed in primary care.

iLFT has increased liver disease diagnosis, improved quality of care and reduced unnecessary secondary care referrals.

\section{OTU-23 INCREASING ATTENDANCE AND ENGAGEMENT OF SUBSTANCE MISUSE CLIENTS WITH HEPATITIS C}

${ }^{1,2}{ }^{2}$ Clare Phillips*, ${ }^{2}$ Abdulkani Yusuf. ${ }^{1}$ Brighton and Sussex University Hospitals NHS Trust, Brighton, UK; ${ }^{2}$ East Sussex Healthcare NHS Trust, Eastbourne, UK

\subsection{6/gutjnl-2019-BSGAbstracts.428}

Introduction In June 2017, a hepatitis C (HCV) clinic was established in the local substance misuse service (SMS) as an outreach from the local district general hospital. The clinic initially operated one afternoon a month, but due to demand was increased to fortnightly in March 2018. The objective of the clinic was to provide HCV care (including work-up and treatment) to clients, who, because of their substance use, might not attend hospital clinics. The aim of this study was to review whether the SMS clinic improved attendance and engagement among these clients compared to the hospital setting.

Methods The outreach clinic database was used to identify all individuals who were offered an appointment in the outreach clinic between June 2017 and December 2018. The hospital electronic booking system was then used to review all historic appointments booked for each of these patients at the hospital. Attendance and engagement with the hospital clinic and the outreach clinic were compared.

Results 51 separate individuals were referred to the SMS clinic between June 2017 and December 2018. 31 of these (61\%) had, at some point (dating as far back as the early 2000s), been referred to the hospital gastroenterology service for their HCV. Of those referred, 12 (39\%) had never attended an appointment. $18(58 \%)$ attended at least once but were subsequently lost to follow-up. 1 (3\%) patient was started on HCV treatment at the hospital but transferred to the SMS clinic once it was established. By comparison, of the 51 referred to the SMS clinic, 17 (33\%) never attended an appointment, 12 (24\%) came at least once but were then lost to follow-up and $22(43 \%)$ remain engaged with the SMS clinic. Of the 22 engaged in the service; 5 have places to start treatment in the next few weeks, 7 have completed treatment and 4 are currently on treatment. 1 patient, lost to follow-up in the SMS clinic, was later treated in prison.

Conclusions Our study shows that, compared to a hospital setting, an outreach clinic at an SMS substantially improves attendance and engagement with care in this cohort of clients with HCV infection. There are a number of factors that may have contributed to the increase in attendance and engagement with the SMS clinic. SMS-based clinics have been shown to reduce barriers to HCV care for SMS attendees (Harris, 2017). We also believe that as our SMS service is based in the centre of town it makes it more accessible for clients compared to the local hospital which is on the peripheries of the town. Finally, an increased awareness of the new, highly effective direct acting antiviral (DAAs) for treatment of HCV might have improved acceptance of testing and treatment in this cohort.

\section{OTU-24 APPETITE FOR IMPROVEMENT; 50\% REDUCTION IN BLOODSTREAM INFECTIONS IN CENTRAL VENOUS CATHETER DELIVERED PARENTERAL NUTRITION}

Margaret Collins*, AJ di Mambro. Gloucestershire Hospitals NHSFT, Cheltenham and Gloucester, UK

\subsection{6/gutjnl-2019-BSGAbstracts.429}

Introduction Bloodstream infection (BSI) is a recognised risk with parenteral nutrition (PN) administered via a central venous catheter $(\mathrm{CVC})^{1}$. It can be life-threatening, leads to an extended hospital stay and, for patients reliant on PN, can mean -4 days without nutrition. NICE estimate the cost of each catheter-related BSI is $£ 9,900^{2}$.

Methods Quality improvement methodologies and tools were used to identify the problem, plan and test change ideas and measure improvements. BSI rates were recorded per 1000 days of PN to allow fair comparison of monthly BSI rates. The amount of PN administered per month was $8-16$ days. A root cause analysis tool (RCA) was developed to investigate each BSI thought related to a CVC. RCA outcomes were reported to the relevant ward manager to highlight areas for improvement and guide action planning. Findings from the RCAs were plotted on a Pareto chart to identify the most frequently occurring factors on which to focus improvements.

A driver diagram was used to plan the improvement process and identify change ideas. Engaging ward nurses, improving their knowledge and understanding of risk factors for developing a BSI and promoting best practice in management of CVCs were key aspects of this project.

1) Actions: Pop-up ward based teaching for nursing staff to highlight the risk factors for BSI and clarify best practice for management of CVC and PN. Nurses completed an anonymous questionnaire to gauge knowledge of $\mathrm{PN}$ and care of a CVC. Their responses guided teaching topics and content of posters to highlight best practice in CVC care.

2) Switch to single, rather than dual, lumen peripherally inserted central catheters (PICCs) to reduce the number of times the PICC was accessed.

3) For in patients requiring $\mathrm{PN}$ for more than 28 days, we trialled the use of a protective cleaning cap on the CVC lumen3.

4) Nutrition support team involvement in mandatory teaching sessions for F1 training and in Trust induction for safe CVC access in $\mathrm{PN}$ patients were also implemented.

Results A 50\% reduction in BSI rates in 2018 compared to 2017.

Conclusions RCA findings show that a number of factors contributed to our patients developing a BSI. Various strategies were used to improve BSI rates. The use of quality improvement tools and methodologies in all stages of this project was key to its success. Future work - develop a PN e-learning module - ward-based CVC update training This quality improvement project has focused on BSI in patients receiving PN however it could be applied to improving the care and use of all CVCs in our Trust. (1) Pironi L, Arends J, Bozzetti F, Cuerda C, Gillanders L, Jeppesen PB, Jolly F, Kelly D, Lal S, Staun M, Szczepanek K, Van Gossum A, Wanten G, Schneider SM, ESPEN Guidelines on chronic intestinal failure in adults. Clinical Nutrition. 2016;35(2): 24-07. (2) NICE. 